\title{
Speeding-Up the K-Means Clustering Method: A Prototype Based Approach
}

\author{
T. Hitendra Sarma and P. Viswanath \\ Pattern Recognition Research Laboratory \\ NRI Institute of Tehnology, Guntur-522009, A.P., India \\ \{t.hitendrasarma, viswanath.pulabaigari\}@gmail.com
}

\begin{abstract}
The paper is about speeding-up the k-means clustering method which processes the data in a faster pace, but produces the same clustering result as the k-means method. We present a prototype based method for this where prototypes are derived using the leaders clustering method. Along with prototypes called leaders some additional information is also preserved which enables in deriving the $k$ means. Experimental study is done to compare the proposed method with recent similar methods which are mainly based on building an index over the data-set.
\end{abstract}

\section{Introduction}

k-means clustering method, for a given data-set, finds $k$ patterns called the $k$ centers (or $k$ means) which represents the $k$ clusters. The centers need to be found such that the sum of squared distances from each data point to its nearest center is minimized. An iterative procedure to find the $k$ centers is given by Lloyd 4 and this is what conventionally called the k-means clustering algorithm. There are several approximate methods like single pass $k$-means method [2] which scans the data-set only once to produce the clustering result. Other approximate methods are to keep important points in the buffer (primary memory) while discarding unimportant points as done by Bradley et. al. [1]. There are other algorithmic approaches which speeds-up the k-means method without compromising with the the quality of the final result. These methods are primarily based on building an index like data structure over the data-set which speeds-up the nearest neighbor finding process. Using a data structure similar to $k d$-tree to speed-up the process was given by Kanungo et al. [3] who gave a filtering algorithm to reduce the number of centers to be searched for a set of points which are enclosed in a hyperrectangle. It proposes a variation of the $\mathrm{kd}$-tree called balanced box-decomposition tree (BBD-tree) and under certain favoring conditions, like clusters being well separated, proves that the total running time of the k-means algorithm using their filtering approach is $O\left(d n \log n+2^{d} m k \log n\right)$, where $n$ is the data-set size, $d$ is the dimensionality of data, $m$ is the number of iterations taken by the $\mathrm{k}$-means algorithm, and $k$ is the number of clusters derived. Clearly, the filtering approach is not a good choice for high dimensional data-sets.

This paper proposes to use only a few selected prototypes from the dataset along with some additional information. Prototypes are selected by using

S. Chaudhury et al. (Eds.): PReMI 2009, LNCS 5909, pp. 56-61 2009.

(C) Springer-Verlag Berlin Heidelberg 2009 
a fast clustering method called the leaders clustering method [5]. Along with leaders additional information like number of patterns and linear sum of patterns that are grouped with a leader are preserved. Recently this kind of approach is adopted to devise approximate hybrid density based clustering methods like rough-DBSCAN 7 and to improve prototype based classification methods like rough fuzzy weighted $k$-nearest leader classifier [6].

The proposed method called leader-k-means (lk-means) clustering method runs in two stages. In the first stage it applies the k-means method over the leaders set. The second stage checks for correctness of the results and if needed applies a correcting step to guarantee that the final clustering result is same as that would have obtained if the k-means method is applied over the entire data-set.

The paper is organized as follows. Section 2 briefly reviews the k-means clustering method while Section 3 reviews the leaders clustering method along with a modified leaders method called modified-leaders to derive leaders along with the number of patterns that are grouped with it, etc. The proposed hybrid method $l k$-means clustering method is described in Section 4. Experimental results are given in Section 5 and Section 6 gives some of the conclusions.

\section{K-Means Clustering Algorithm}

$\mathrm{k}$-means clustering method is a partition based method and each cluster is represented by its centroid (mean). Let $\mathcal{D}=\left\{x_{1}, x_{2}, \ldots, x_{n}\right\}$ be the dataset of dimensionality $d$. The means has to be found such that the criterion $J=\sum_{i=1}^{n}\left\|x_{i}-m\left(x_{i}\right)\right\|^{2}$ is minimized, where $m\left(x_{i}\right)$ is the nearest mean to $x_{i}$. The iterative procedure given by Lloyd 4 to find the $k$ means is given in the Algorithm 1,

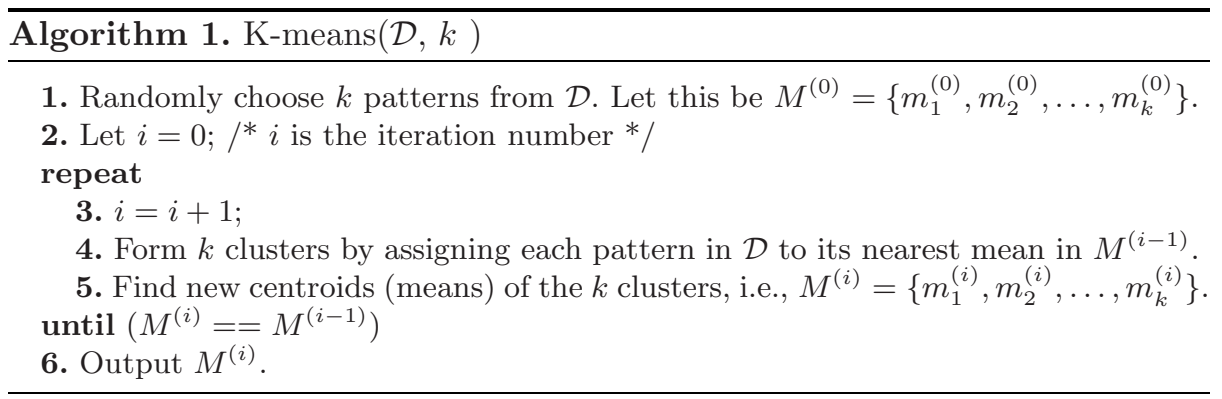

\section{Leaders Clustering Method}

In leaders clustering method each cluster is represented by a pattern called leader and all other patterns in the cluster are its followers. For a given threshold distance $\tau$, leaders method maintains a set of leaders $\mathcal{L}$, which is initially empty 
and is incrementally built. For each pattern $x$ in the data set $\mathcal{D}$, if there is a leader $l \in \mathcal{L}$ such that distance between $x$ and $l$ is less than or equal to $\tau$, then $x$ is assigned to the cluster represented by $l$. Otherwise $x$ itself becomes new leader and is added to $\mathcal{L}$. The algorithm outputs the set of leaders $\mathcal{L}$.

The leaders method is modified in-order to be used with k-means method. The modifications are, (i) to replace a leader by its cluster centroid (so, a leader is no more a pattern in the data-set, but all its followers are patterns from the data-set), (ii) to store along with leaders, a count, which is the number of patterns that are present in its cluster, and (iii) linear sum of all followers. The modified leaders method is given in Algorithm 2. The output of the method is leaders set along with count, linear sum, and also the data-set rearranged and stored according to the clusters.

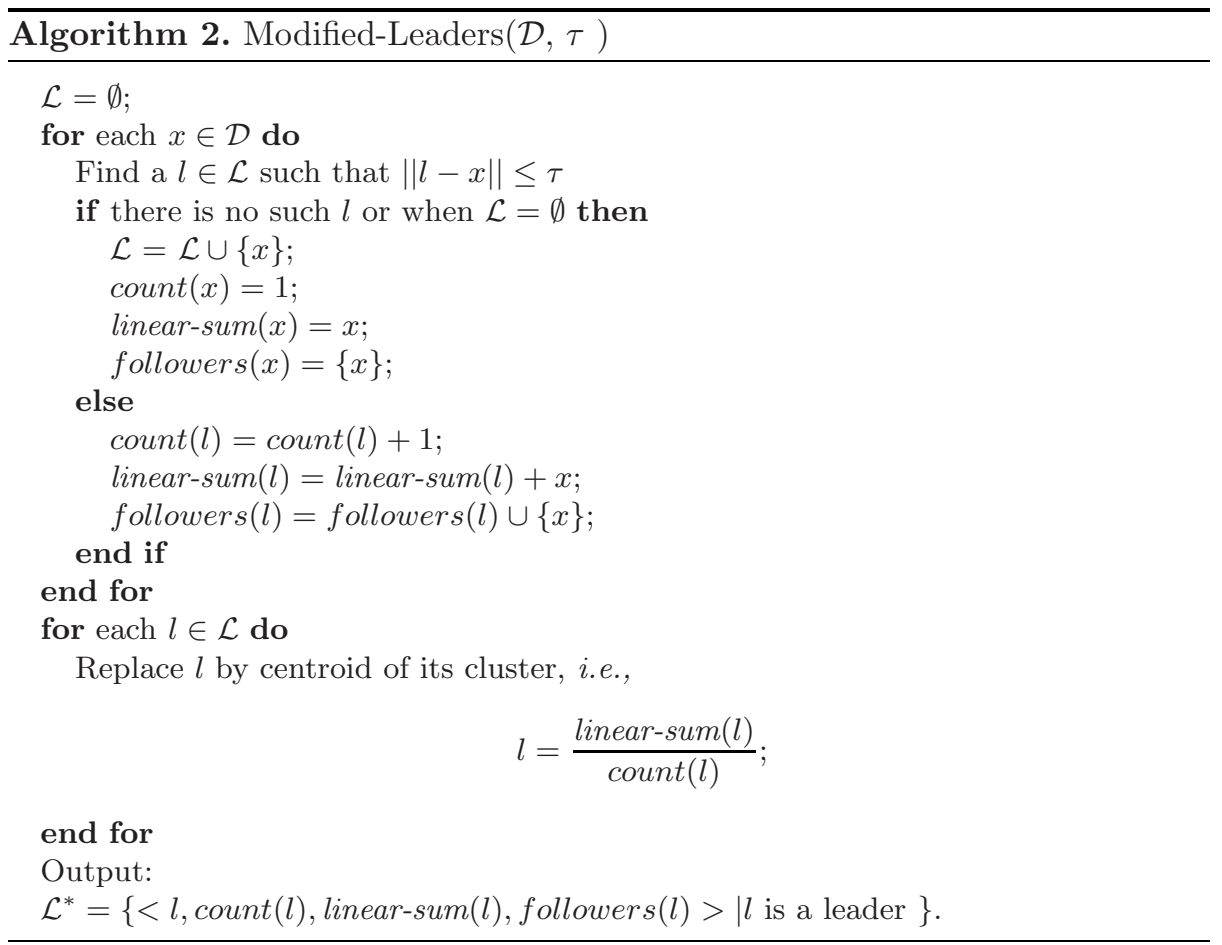

\section{$4 \quad l k$-Means: Leader Based K-Means Clustering Method}

The proposed method $l k$-means clustering method runs in two stages. The first stage called $l k$-means-first-stage basically runs the Lloyd's $k$-means method, but using leaders set, their count and linear-sum values. The method is iterated till it converges. The second stage is called $l k$-means-correcting-stage which checks for correctness of the results and if needed applies the correcting step so that 
the final result is same as that obtained by applying(including itself) the Lloyd's algorithm using the entire data-set.

The first step called $l k$-means-first-stage is same as Lloyd's k-means as given in Algorithm 1, but is applied using the leaders set (instead of the entire data-set). The method starts with initially chosen random patterns from the data-set as its seed-points, and in each iteration, each leader is assigned to the nearest mean pattern to form the $k$ clusters of leaders. The new means (i.e., the new centroids) of each cluster (cluster of leaders) is found as explained. Let $l_{1}, l_{2}, \ldots, l_{p}$ be the leaders in a cluster. Then its new centroid is $\frac{\sum_{j=1}^{p} \operatorname{linear}-\operatorname{sum}\left(l_{j}\right)}{\sum_{j=1}^{p} \operatorname{count}\left(l_{j}\right)}$. The method is iterated till it converges. The clustering result of this stage consists clusters of leaders. By replacing each leader by the set of its followers, we get a partition of the data-set $\mathcal{D}$. Let this partition be $\pi^{l}$, and that obtained by employing Algorithm 1 (i.e., the original k-means method) over the entire data-set (keeping the seed points same) be $\pi$. Now, $\pi^{l}$ need not be same as $\pi$, because there may be a leader $l$ which is assigned to a mean $m_{i}$ (according to $l k$-means-first-stage), but a follower of $l$ may be actually closer to some other mean $m_{j}$ such that $m_{i} \neq m_{j}$. Let the follower be $x_{f}$. So, this situation arises when $\left\|l-m_{i}\right\|<\left\|l-m_{j}\right\|$, but $\left\|x_{f}-m_{i}\right\|>\left\|x_{f}-m_{j}\right\|$. The pattern $x_{f}$ according to the original $k$-means

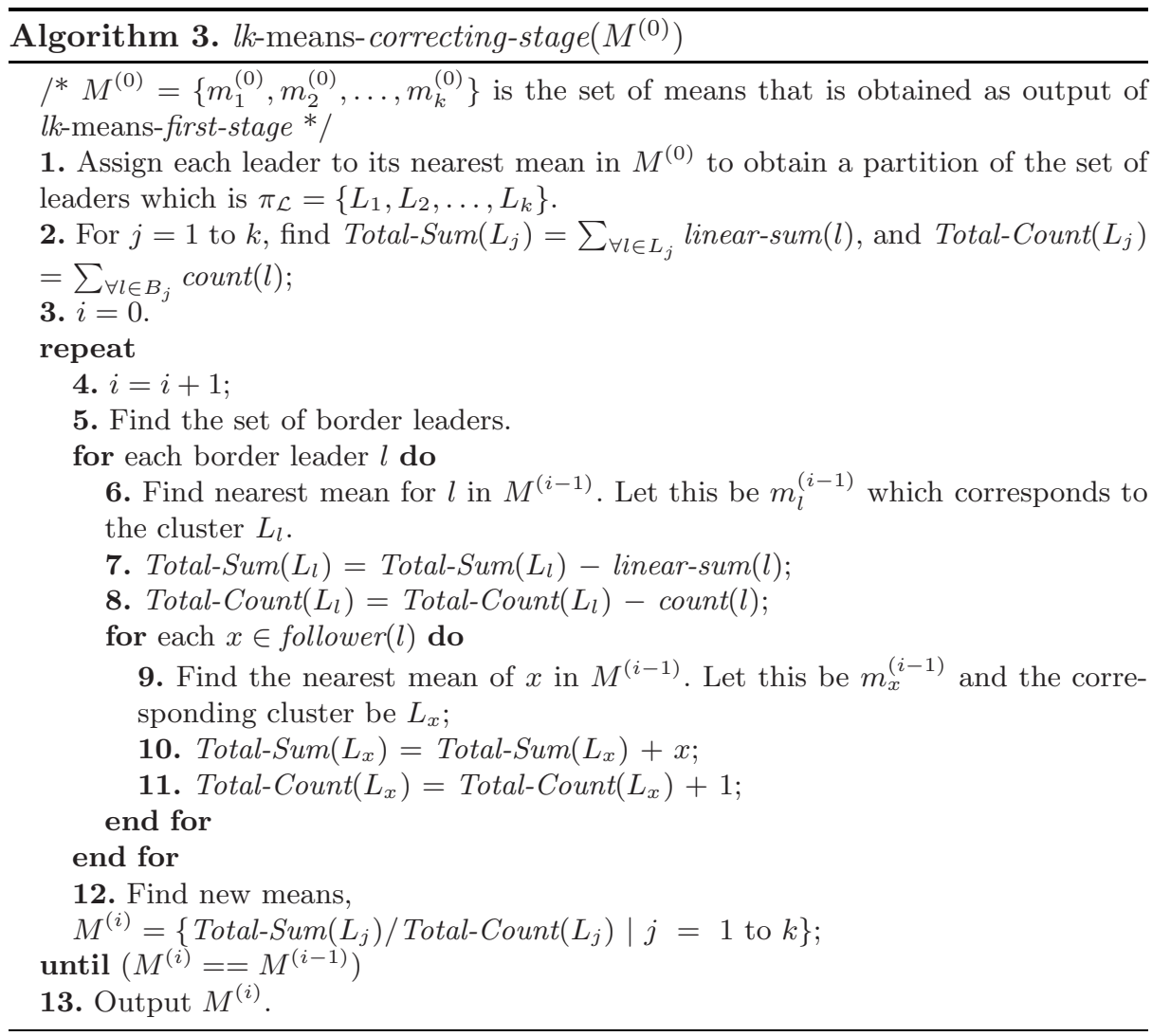


method should be included with the cluster for which $m_{j}$ is the mean, but $l k$-means-first-stage assigned this to the mean $m_{i}$. Such leaders are named as boarder-leaders. The set of such border-leaders can be found as part of last iteration of the method $l k$-means-first-stage. The second stage is called $l k$-meanscorrecting-stage. If the set of border leaders is non-empty, then $\pi^{l}$ and $\pi$ need not be same. A correcting step is applied over the result of $l k$-means-first-stage in order to get the same clustering result as that would have obtained by using the original Lloyd's algorithm (Algorithm 1). Only the followers of border leaders are reconsidered in the correcting stage as given in Algorithm 3 . Each border leader $l$ is removed from its cluster and each follower of this leader $l$ is reconsidered as an individual pattern and assigned to its nearest mean. The process is repeated till convergence.

\section{$5 \quad$ Experimental Results}

Experiments are done with (i) the Pendigits data-set available at the UCI Machine Learning Repository, and (ii) a series of synthetic data-sets of varying dimensionality. 39 different synthetic data-sets of dimensionality ranging from 2 to 40 are generated as follows. Each data-set has 60000 patterns. Each dataset is generated from a tri-modal Gaussian distribution $p(x)=\frac{1}{3} N\left(\mu_{1}, \Sigma_{1}\right)+$ $\frac{1}{3} N\left(\mu_{2}, \Sigma_{2}\right)+\frac{1}{3} N\left(\mu_{3}, \Sigma_{3}\right)$. For dimensionality $2, \mu_{1}=(0,0), \mu_{2}=(5,5), \mu_{3}=$ $(-5,5)$; for dimensionality $3, \mu_{1}=(0,0,0), \mu_{2}=(5,5,5), \mu_{3}=(-5,5,-5)$; and so on upto dimensionality 40. In Each case the covariance matrix is taken as the Identity matrix of size $d \times d$, where $d$ is the dimensionality of the data.

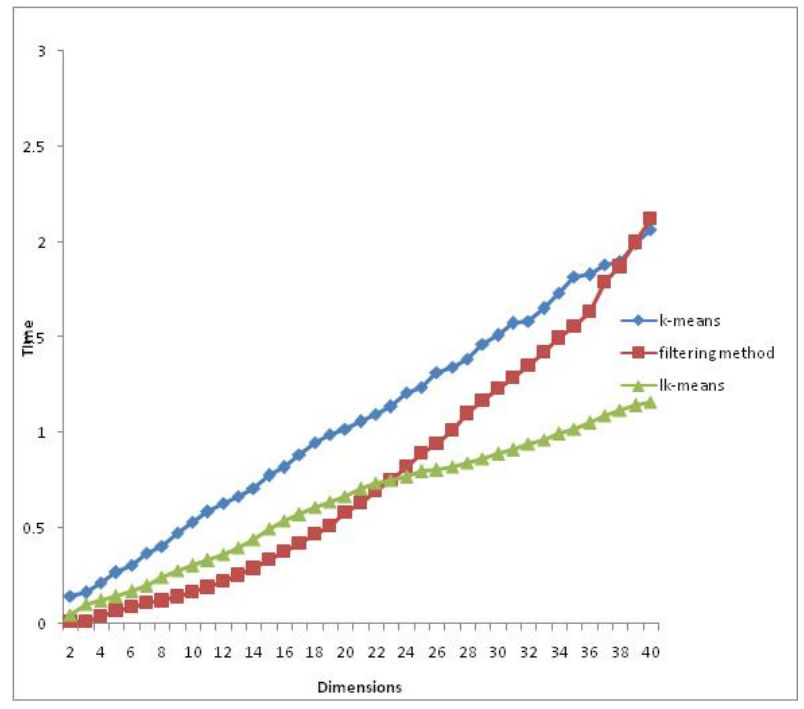

Fig. 1. Comparison of time taken by various methods 
For Pendigits data-set $k$ value taken is 10 , and for synthetic data-sets it is 3 . The parameter $\tau$ used to generate the set of leaders is taken as $5 \%$ of the average distance between a pair of distinct random patterns over 10 random trials.

For Pendigits data-set, the k-means method (Algorithm 1) has taken 0.463 seconds, whereas the filtering approach has taken time equal to 0.326 seconds. But the proposed method has taken time equal to 0.294 seconds only. This is because, for the Pendigits data, its dimensionality is 16, and filtering approach can work well only for low dimensional data-sets. This point is amplified by the synthetic data-sets of varying dimensionality, which clearly shows that the filtering approach can reduce the time requirement considerably for low dimensional data-sets, but fails to do so for high dimensional data-sets. See Figure 1

\section{Conclusions}

The paper presented a hybrid method to speed-up the k-means clustering method. The proposed method scales well for large and high dimensional data-sets, in contrast to other approaches which are not suitable to work with high dimensional data-sets. The proposed method is a prototype based one, where the prototypes called leaders are derived from the data-set using the leaders clustering method.

\section{References}

1. Bradley, P.S., Fayyad, U., Raina, C.: Scaling clustering algorithms to large databases. In: Proceedings of Fourth International Conference on Knowledge Discovery and Data Mining, pp. 9-15. AAAI Press, Menlo Park (1998)

2. Jain, A.K., Murty, M.N., Flynn, P.J.: Data clustering: A review. ACM Computing Surveys 31(3), 264-323 (1999)

3. Kanungo, T., Mount, D.M., Netanyahu, N.S.: An efficient k-means clustering algorithm: Analysis and implementation. IEEE Transactions on Pattern Analysis and Machine Intelligence 24(7), 881-892 (2002)

4. Lloyd, S.P.: Least squares quantization in PCM. IEEE Transactions on Information Theory 28, 129-137 (1982)

5. Spath, H.: Cluster Analysis Algorithms for Data Reduction and Classification. Ellis Horwood, Chichester (1980)

6. Babu, V.S., Viswanath, P.: Rough-fuzzy weighted k-nearest leader classifier for large data sets. Pattern Recognition 42, 1719-1731 (2009)

7. Viswanath, P., Suresh Babu, V.: Rough-DBSCAN: A fast hybrid density based clustering mehtod for large data sets. Pattern Recognition latters (2009) doi:10.1016/j.patrec.2009.08.008 